\title{
An approach to the measurement of the nonlinear refractive index of very short lengths of optical fibers
}

\author{
E. Rivera-Pérez, A. Carrascosa, A. Díez , E. P. Alcusa-Sáez, M. V. Andrés \\ Departamento de Física Aplicada y Electromagnetismo-ICMUV, Dr. Moliner 50, \\ 46100 Burjassot, Spain \\ *antonio.diez@uv.es
}

\begin{abstract}
:
A method for the measurement of the nonlinear-refractive index coefficient in single-mode optical fibers is presented. It takes advantage of the high sensitivity of the acousto-optic interaction effect in optical fibers to the fiber properties. Direct measurement of the nonlinear-refractive index change resulting from cross-phase modulation between a probe and a pump signal is obtained from the fiber's acousto-optic interaction performance. It is a non-interferometric method in which a very short length of fiber $(<0.25$ $\mathrm{m})$ is required.
\end{abstract}


Optical fiber nonlinearity is exploited in several real applications, as supercontinuum light sources [1], and some techniques for ultrashort pulse compression [2]. Despite the nonlinear coefficient of silica is quite low compared to other glasses, nonlinearities in silica-based optical fibers may cause substantial effects, mainly because of the tight light confinement at the core, and the possibility of long propagation distances.

A wide variety of methods for measuring the nonlinear-index coefficient, $\mathrm{n}_{2}$, in optical fibers have been reported, in which a number of different strategies have been exploited. Among others, we can mention those based on the analysis of the spectral features generated by self-phase modulation [3, 4], on the direct measurement of the nonlinear phase shift using interferometric methods [5, 6], or those based on nonlinear-polarization evolution [7]. Most of previously reported methods require the use of long sections of the fiber under test (FUT), typically of hundreds of meters, which implies, in practice, that fiber attenuation and polarization issues must be taken into account. Moreover, they provide an average value of the nonlinearity over the entire fiber length. The development of methods that can perform the measurement with short sections of fiber is desired, and particularly interesting for the characterization of novel fibers that can be expensive and/or difficult to fabricate. As far as we know, there is only one method, the induced grating autocorrelation (IGA) technique, that has been applied to measure $\mathrm{n}_{2}$ using lengths of fibers in the scale of meters [8]. IGA technique requires a rather complicated experimental arrangement. In particular, for highly nonlinear photonics crystal fibers (PCFs) that exhibit quite large nonlinear coefficients, IGA measurements were demonstrated with fiber sections in the order of one meter [9].

Over the years, the acousto-optic (AO) interaction effect in optical fibers has been exploited for the development of dynamic, tunable and reconfigurable all-fiber devices [10]. In the last years, it has been proposed as a highly sensitive tool, particularly suited for fine characterization of optical fibers [11-13]. Measurement of fiber radius fluctuations in the nm scale, and core refractive index variations in the order of $10^{-8}$ in single-mode fibers were reported [12]. Such outstanding sensitivity relies on (a) the high sensitivity of the AO phase-matching condition with the structural and material properties of the fiber, and (b) on the possibility of using long AO interaction lengths (up to some tens of $\mathrm{cm}$ ) that leads to the generation of narrow bandwidth (even sub-nm) coupling notches.

Here, we propose and demonstrate an experimental method for investigating the optical nonlinearity of single-mode optical fibers, which is based on the AO interaction phenomenon using flexural elastic waves. In this technique, very short lengths of fiber, typically of few tens of $\mathrm{cm}$, are required, which allows the use of this method for the characterization of nonlinear properties of non-conventional fibers, as for example, microstructured optical fibers.

A flexural elastic wave that propagates along a single-mode optical fiber creates a periodic refractive index perturbation (a travelling "acoustic" long-period grating) that can cause coupling between the $\mathrm{LP}_{01}$ core mode and copropagating $\mathrm{LP}_{1 \mathrm{~m}}(\mathrm{~m}=1,2,3 \ldots)$ cladding modes. Coupling to a given cladding mode causes a notch in the spectrum of the light transmitted through the fiber $[9,13]$. The notch is centered at the Bragg wavelength $\lambda_{R}=\Delta \mathrm{n}_{\text {eff }} \Lambda$, where $\Delta \mathrm{n}_{\text {eff }}$ is the effective index difference between the core mode and the cladding mode and $\Lambda$ is the period of the acoustic wave in the fiber. 
The characteristic features of the notch and, specifically, the resonance wavelength, are determined by the optical fiber properties. A small change of refractive index at the core area will cause the shift of the notch since it affects the effective indices of the optical modes, particularly, the effective index of the core mode. In the experimental realization, changes of refractive index were produced by an optical pump pulse, which was delivered to the fiber under test where AO interaction was taking place. The intensitydependent effective index change of the $\mathrm{LP}_{01}$ core mode, $\Delta \mathrm{n}_{01}$, can be obtained through the interrogation of the AO notch. This was done by using a low power probe laser tuned at the mid-point of the notch edge, where the transmittance $\mathrm{T}$ depends linearly with the optical wavelength [12]. The refractive index change caused by the pump pulse produces a change in the transmittance, $\Delta \mathrm{T}$, of the probe signal. For a small index perturbation, $\Delta \mathrm{T}$ can be approximated by a first order Taylor series expansion as,

$$
\Delta T \approx\left[\frac{\partial T}{\partial \lambda}\right] \times\left[\left(\frac{\partial}{\partial \lambda} \Delta n_{e f f}-\frac{\Delta n_{e f f}}{\lambda}\right)\right]^{-1} \Delta n_{01}
$$

In the derivation of Eq. (1), the change of effective index of the cladding mode produced by the pump pulse is neglected since the sensitivity in most fibers of the effective index of low-order $\mathrm{LP}_{1 \mathrm{~m}}$ cladding modes to changes of core index is few orders of magnitude lower than that of the core mode. In Eq. (1) the first bracket is just the slope of the transmittance of the AO notch at the probe wavelength, which can be obtained simply from the spectrum. The second bracket involves the optical wavelength $\lambda$, and the properties of the fiber modes that can be obtained either from theoretical modelling or, experimentally from a fiber acousto-optic characterization experiment, as it is shown later. Therefore, using Eq. (1), the intensity-dependent effective index change of the core mode $\Delta \mathrm{n}_{01}$ can be obtained from measurements of the probe transmittance changes that result from the propagation of the pump pulse.

We performed the experiments with a commercial step-index fiber (SM980 from Fibercore Ltd, nominal $\mathrm{NA}=0.15$, and $\lambda_{\text {cutoff }}=920 \mathrm{~nm}$ ). We used a fiber length of just $20 \mathrm{~cm}$. In a first experiment, we carried out the characterization of the acousto-optic interaction effect in this fiber at the optical wavelength range of the probe laser. Vibrations were generated by a piezoelectric transducer driven by a radio-frequency (RF) signal generator, and were coupled to the fiber with a horn. The fiber polymer coating was stripped out to avoid the attenuation of the acoustic wave and the fiber was held straight. The tuning response of the first four notches is shown in Fig. 1(a).

From the measurements given in Fig. 1(a), it is possible to obtain the core-cladding modal effective index difference, $\Delta \mathrm{n}_{\text {eff }}$, as a function of the optical wavelength by using the Bragg wavelength condition. For this, it is necessary to know the dispersion relation of the flexural elastic mode in the fiber, which was measured experimentally using an optical vibrometer [13]. Figure 1 (b) shows the experimental results for the four resonances, along with theoretical calculations. The theoretical calculations were performed by solving rigorously the Maxwell equations with the corresponding boundary conditions, assuming a threelayer step-index model (core/clad/air) for the fiber, and taking into account the glass refractive index dispersion. The best fit was obtained for a step-index fiber with $\mathrm{NA}=0.15, \lambda_{\text {cutoff }}=933 \mathrm{~nm}$, and fiber diameter $=125 \mu \mathrm{m}$. From the experimental result shown in Fig. 1(b), we can obtain the effective index difference $\Delta \mathrm{n}_{\text {eff }}$ at the probe wavelength, as well as the derivative of $\Delta \mathrm{n}_{\text {eff }}$ against the optical wavelength, required to evaluate Eq. (1). 

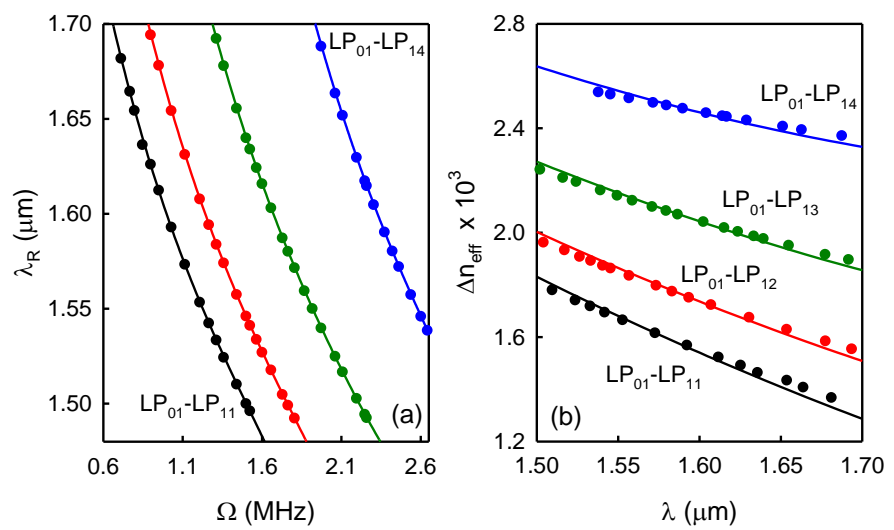

Fig. 1. (a) Resonance wavelength as a function of the acoustic frequency. Dots are experimental data and solid lines are just a guide to the eye. (b) Effective index difference between the $\mathrm{LP}_{01}$ core mode and $\mathrm{LP}_{1 \mathrm{~m}}$ cladding mode, as a function of the optical wavelength. Dots are experimental data and lines are theoretical calculations from a three-layer step-index $\operatorname{model}\left(\mathrm{NA}=0.15, \lambda_{\text {cutoff }}=933 \mathrm{~nm}\right.$, and fiber diameter $\left.=125 \mu \mathrm{m}\right)$.

Figure 2 shows the experimental arrangement used for the measurement of the nonlinear refractive index. $\mathrm{AO}$ interaction was produced in the fiber as described above, generating a notch at the $\mathrm{C}$ band. Optical pump pulses were delivered to the fiber section where the acoustic grating was generated. The pump pulses were generated by a MOPA laser system consisting of an amplitude modulated, linearly polarized diode laser emitting at $1064 \mathrm{~nm}$ that was power amplified by a double-clad polarization maintaining Yb-doped fiber amplifier. The pump system was able to emit linearly polarized pulses (PER $>30 \mathrm{~dB}$ ) of about $30 \mathrm{~ns}$ duration, up to $30 \mu \mathrm{J}$ energy, at a repetition rate of few kHz. Fig. 2 (b) shows the temporal profile of a typical pump pulse. Care was taken to measure accurately the pulse temporal profile, with a $5 \mathrm{GHz}$ bandwidth photodetector and a fast oscilloscope. The pump laser was launched into the FUT using an aspheric lens. The use of ns pump pulses instead of shorter ps pulses was preferred to ensure that possible chirping effects produced by the pump pulse on the acoustic grating can be neglected. Pump pulse energy was measured with a calibrated power meter (Ophir NOVA II, PE9-C).

The probe signal used to analyze the transmittance fluctuations caused by the propagation of the pump pulse was obtained from a CW tunable diode laser emitting linearly polarized light (PER $>30 \mathrm{~dB}$ ) from 1530-1620 nm. The intensity of the probe signal was measured with a fast photodetector and recorded with an oscilloscope.

Two beam combiners/splitters and one filter were used to combine/separate both optical signals. Additionally, a half-wave plate and a fiber polarization controller were inserted to manage the polarization of the pump and probe signals, respectively.

Fig. 2 (c) shows the $\mathrm{AO}$ notch used in the experiments, it results from the coupling between the $\mathrm{LP}_{01}$ core mode and the $\mathrm{LP}_{12}$ cladding mode. The notch is centered at $1573 \mathrm{~nm}$ and the $-3 \mathrm{~dB}$ bandwidth is $1.8 \mathrm{~nm}$. The shift of the AO notch was interrogated by measuring the variations in transmittance of the probe signal that was tuned at two wavelengths, each one lying at the linear part of each notch edge, as indicated in Fig. 2 (c). Figure 3 shows typical transmittance traces of the probe signal when a pump pulse propagated along the FUT. In both cases, transmittance changes were observed only during the time that the pump pulse was travelling across the acoustic grating. When the probe laser was tuned at the short- 
wavelength edge of the notch $\left(\lambda_{\mathrm{p} 1}\right)$, the propagation of the pump pulse produced an increase of transmittance, while a decrease of transmittance was observed when the probe was tuned to the longwavelength edge of the notch $\left(\lambda_{\mathrm{p} 2}\right)$. This behavior confirms the redshift of the notch. As expected, the transmittance change increases as the energy of the pump pulse was increased. The peak transmittance change, $\Delta \mathrm{T}_{\mathrm{p}}$, is obtained when the whole acoustic grating is exposed uniformly to the peak power of the pulse. Notice that the shape and duration of the transmittance change is similar to the temporal profile of the pump pulse. This happens because the pulse extension $(\sim 6 \mathrm{~m})$ is much longer than the AOI interaction region $(20 \mathrm{~cm})$, and because slow contributions, as thermal heating, are negligible in this fiber. We have to notice that when we carried out the same experiment with fibers with higher optical absorption losses, the transmittance trace showed a long trailing tail that results from the refractive index change due to thermal heating that extends to the ms time scale. For such fibers, shorter and less energetic pulses are desirable.
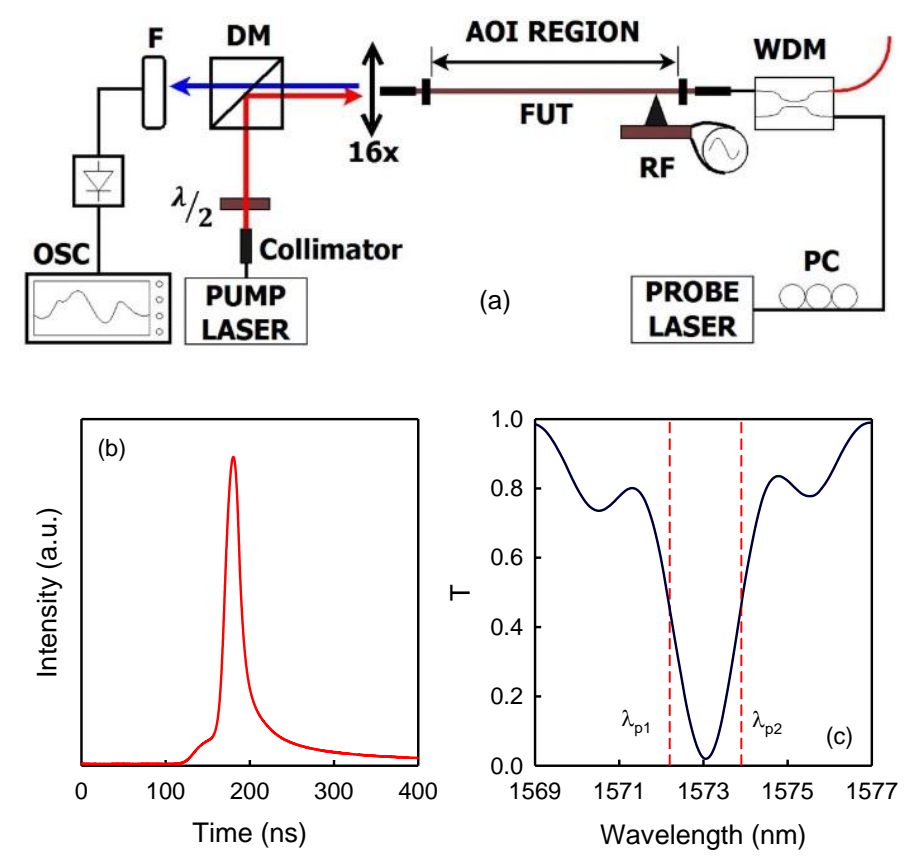

Fig. 2. (a) Experimental arrangement. DM: Dichroic mirror; PC: polarization controller; F: bandpass filter; WDM: wavelength division multiplexer. (b) Temporal profile of a typical pump pulse. (c) Fiber transmittance spectrum showing the AO generated notch used in the experiments. Length of the acoustic grating: $18 \mathrm{~cm}$. RF signal frequency: $1.36 \mathrm{MHz}$. Red dashed lines indicate the two probe wavelengths used for the interrogation of the notch's shift, $\lambda_{\mathrm{p} 1}=1572.2 \mathrm{~nm}$ and $\lambda_{\mathrm{p} 2}=1573.9 \mathrm{~nm}$.

The probe transmittance change depends strongly on the polarization state of the pump and probe beams since the increase of refractive index noticed by the probe light (due to XPM) depends on the polarization of the two beams [6]. Figure 3(c) shows different probe traces obtained for a fixed pump pulse energy and different polarization states of the two beams that were achieved by rotating the halfwave plate and the fiber PC. Substantial variations in amplitude were found, as it can be seen in Fig. 3(c). Curves $\mathrm{i}$ and ii correspond to orientations of half-wave plate and PC for which we obtained the largest and shortest effect. According to the XPM nonlinear theory [15], the largest and shortest refractive index 
change is achieved for linearly polarized beams when the polarization planes of both beams are parallel and orthogonal, respectively. Taking into account that the fiber length used is very short and it was carefully kept straight, we can assume that curves $i$ and ii correspond to the two cases mentioned above.

In other techniques based on XPM for measuring the nonlinear refractive index in which long sections of fiber are required, the polarization drift that typically happens in non-polarization maintaining optical fibers, imposes the use of depolarized light [6]. In such a case, the refractive index change noticed by the probe beam is $2 / 3$ the refractive index change achieved in the optimum polarization combination.

Measurements of the probe transmittance change as a function of the pump pulse energy, $E_{p}$, were performed at the two probe wavelengths. The polarization of the two beams was adjusted to maximize $\Delta T$. Figure 4(a) shows the peak transmittance change, $\Delta T_{p}$, as a function of $E_{p}$. It was observed that $\Delta T_{p}$ increased with the pump pulse energy. For low pulse energies, the increase is linear but the linearity is lost for large energies (not shown in the figure) as the shift of the notch becomes larger and the probe wavelength was no longer tuned within the linear part of the notch edge. Notice that the $\Delta \mathrm{T}_{\mathrm{p}}$ is slightly larger when the probe laser was tuned to the right-hand side edge of the notch, in agreement with the slopes of the notch's edges (see Table I).
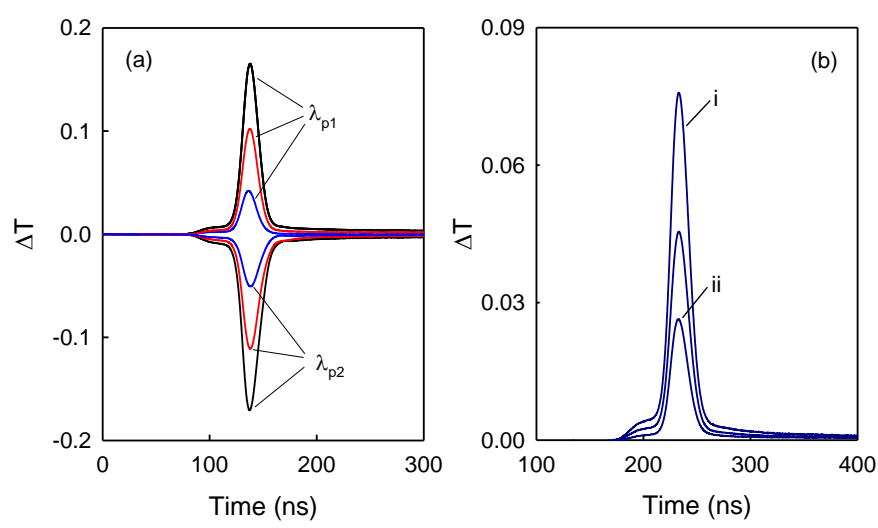

Fig. 3. (a) Transmittance change of the probe laser as a function of time when a pump pulse is delivered to the acoustic grating. Probe laser wavelength: $\lambda_{\mathrm{p} 1}=1572.2 \mathrm{~nm}, \lambda_{\mathrm{p} 2}=1573.9 \mathrm{~nm}$. Different curves correspond to pump pulses of different energies. (b) Transmittance change of the probe laser as a function of time for a fixed pump pulse energy $\left(\mathrm{E}_{\mathrm{p}}=8 \mu \mathrm{J}\right)$ and different polarization states of the pump and probe beams.

The extension of the pump pulse in the fiber is much longer than the acoustic grating. Therefore, when the pump pulse is located at the center of the grating, the intensity distribution of the pump pulse along the grating is essentially constant, so the pump pulse just causes a uniform increase of the average refractive index. Thus, the theoretical bases described above, which are exact for uniform gratings, can be applied without loss of accuracy.

The intensity-dependent effective index change of the probe light, $\Delta \mathrm{n}_{01}$, produced by the pump pulse when it is located at the center of the grating can be obtained from $\Delta \mathrm{T}_{\mathrm{p}}$ using Eq. (1). The different quantities required to evaluate $\Delta \mathrm{n}_{01}$ from the measurements of $\Delta \mathrm{T}_{\mathrm{p}}$ for this experiment are given in Table 1. Figure 4(b) shows $\Delta \mathrm{n}_{01}$ as a function of the pump pulse peak power, $\mathrm{P}_{\mathrm{p}}$, for the two probe wavelengths. 
The peak power of the pump pulses was obtained from the measurement of the pulse energy and taking into account the specific temporal profile of the pulses.

Table 1. Value of the different quantities.

\begin{tabular}{cccc}
\hline$\lambda_{\text {probe }}(\mathrm{nm})$ & $\partial T / \partial \lambda\left(\mathrm{nm}^{-1}\right)$ & $\partial \Delta n_{\text {eff }} / \partial \lambda\left(\mathrm{nm}^{-1}\right)$ & $\Delta n_{\text {eff }}$ \\
\hline 1572.2 & -0.69 & $-2.1935 \times 10^{-6}$ & 0.001796 \\
1573.9 & 0.72 & & \\
\hline
\end{tabular}

In the experiment, the polarization of the beams was adjusted to achieve maximum transmission change. Then, it is reasonable to assume that the pump and probe beams are linearly polarized, with the same polarization plane. Additionally, the peak power of the pump pulses is few orders of magnitude larger than the power of the probe signal. Under these conditions, the intensity-dependent nonlinear index change noticed by the probe light (due to XPM) when the pump pulse is present in the fiber, can be related to the nonlinear-index coefficient, $\mathrm{n}_{2}$, as follows [5],

$$
\Delta n_{01} \approx 2 n_{2}\left(P_{p} / A_{e f f}\right) \quad A_{e f f}=\pi\left(w_{p}^{2}+w_{s}^{2}\right) / 2
$$

where $\mathrm{w}_{\mathrm{s}}$ and $\mathrm{w}_{\mathrm{p}}$ are the probe and pump beam radius. Thus, $\mathrm{n}_{2}$, can be obtained from the slope in Fig. 4(b). For the fiber of the experiments we obtained $\mathrm{n}_{2}=2.53 \times 10^{-20} \mathrm{~W}^{-1} \mathrm{~m}^{2}$, where the values of $\mathrm{w}_{\mathrm{s}}=4.61 \mu \mathrm{m}$ and $\mathrm{w}_{\mathrm{p}}=2.88 \mu \mathrm{m}$ were computed for a step-index fiber with $\mathrm{NA}=0.15, \lambda_{\text {cutoff }}=933 \mathrm{~nm}$.

Measurement of the nonlinear refractive index were also performed using the $\mathrm{LP}_{01}-\mathrm{LP}_{11}$ and the $\mathrm{LP}_{01}$ $\mathrm{LP}_{13} \mathrm{AO}$ notches. The values of $\mathrm{n}_{2}$ obtained in these experiments were $2.48 \times 10^{-20} \mathrm{~W}^{-1} \mathrm{~m}^{2}$ and $2.59 \times 10^{-20}$ $\mathrm{W}^{-1} \mathrm{~m}^{2}$, respectively. The different values of $\mathrm{n}_{2}$ are within a range of variation of about $4 \%$. We believe that such a dispersion of $\mathrm{n}_{2}$ values is due of to the accuracy of the technique.

The value of $\mathrm{n}_{2}$ obtained for the fiber SM980 is compatible with previous reports for similar fibers. In [16], a value of $\mathrm{n}_{2}=2.72$ is reported for a similar fiber (not identical) with similar $\mathrm{GeO}_{2}$ concentration, which is about $7 \%$ larger than the value of $\mathrm{n}_{2}$ measured with our method. We must point out that the measured values of $\mathrm{n}_{2}$ have a typical error of $5 \%$, they are affected by the experimental technique used to measure them, and the values reported in the bibliography exhibit a quite large scatter, even when the same fiber samples are characterized [17]. A fruitful discussion about this issue can be found in [15].

The main source of error comes from the measurement of the pump pulse energy, the peak power estimation, and on the computation of the effective area. Additionally, it is worth to mention that the value of $\mathrm{n}_{2}$ obtained from these experiments includes the electronic contribution to the nonlinearity of the glass but also a contribution due to electrostriction [18]. The use of shorter pump pulses would eventually allow reducing the contribution to the overall nonlinearity due to electrostriction. However, the retrieval of the nonlinear refractive index from the transmittance traces requires a more involved theoretical analysis due to the chirping effects induced by a pump pulse shorter than the acoustic grating itself. 

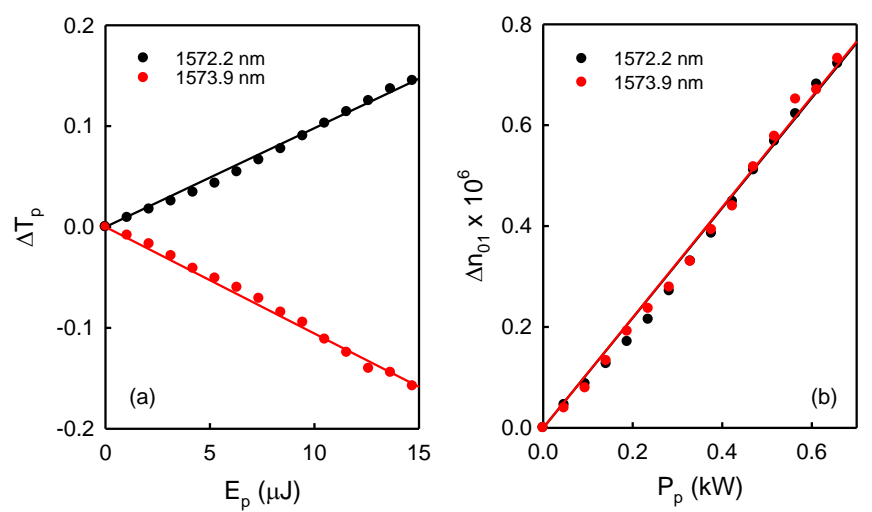

Fig. 4. (a) Peak transmittance change as a function of the pump pulse energy, for the two probe wavelengths.

(b) Intensity-dependent effective index change of the $\mathrm{LP}_{01}$ mode as a function of pump peak power.

In summary, we have shown that acousto-optic interaction effect in optical fibers can be exploited to investigate the nonlinear refractive index in single-mode optical fibers. The technique is based on the high sensitivity of the AO resonance wavelength to small changes of the fiber refractive index. Unlike most previously reported methods that require the use of long sections of fiber, typically hundreds of meters or even few $\mathrm{km}$, we performed our experiments with just $20 \mathrm{~cm}$ of fiber, which allows the characterization of novel fibers that eventually can be expensive and/or difficult to fabricate. The value of $n_{2}$ obtained for the fiber analyzed is compatible with previous reports.

\section{Acknowledgements}

The authors acknowledge the Ministerio de Economía y Competitividad (MINECO) and Fonds Européen de Développement Économique et Régional (FEDER) (TEC2016-76664-C2-1-R); Generalitat Valenciana (PROMETEOII /2014/072).

\section{References}

1. J. M. Dudley, J. R. Taylor, Supercontinuum generation in optical fibers, (Cambridge University Press, 2010).

2. B. Nikolaus and D. Grischkowsky, Appl. Phys. Lett., 42, 1 (1983).

3. R. H. Stolen and C. Lin, Phys. Rev. A., 17, 1448 (1978).

4. A. Boskovic, S. V. Chernikov, J. R. Taylor, L. Gruner-Nielsen, and O. A. Levring, Opt. Lett. 21, 1966 (1996).

5. M. Monerie and Y. Durteste, Electron. Lett., 23, 961 (1987).

6. T. Kato, Y. Suetsugu, M. Takagi, E. Sasaoka, and M. Nishimura, Opt. Lett. 20, 988 (1995).

7. J. Subías, J. Pelayo, R. Alonso, F. Villuendas, C. Heras, , J. Opt. Soc. Am. B, 19, 390 (2002).

8. H. Garcia, A. M. Johnson, F. A. Oguama, and S. Trivedi, Opt. Lett. 28, 1796-1798 (2003)

9. R. Kuis, A. Johnson, and S. Trivedi, Opt. Express 19, 1755 (2011).

10. K. S. Hong, H. C. Park, B. Y. Kim, I. K. Hwang, W. Jin, J. Ju, D. I. Yeom, Appl. Phys. Lett. 92, 031110 (2008).

11. E. P. Alcusa-Sáez, A. Díez, M. González-Herráez, and M. V. Andrés, Opt. Lett. 39, 1437 (2014).

12. E. P. Alcusa-Sáez, A. Díez, M. González-Herráez, and M. V. Andrés, Opt. Express 23, 7345 (2015). 
13. E. P. Alcusa-Sáez, A. Díez, and M. V. Andrés, Opt. Express 24, 4899 (2016).

14. H. S. Kim, S. H. Yun, I. K. Kwang, and B. Y. Kim, Opt. Lett. 22, 1476 (1997).

15. G. Agrawal, Nonlinear Fiber Optics, (Academic Press, 2013).

16. A. Wada, S. Okude, T. Sakai, and R. Yamauchi, Electronics and Communications in Japan (Part I Communications) 79, 12 (1996).

17. A. Fellegara, M. Artiglia, S. B. Andreasen, A. Melloni, F. P. Espunes, and S. Wabnitz, Electron. Lett. 33, 1168 (1997).

18. E. L. Buckland, R. W. Boyd, Opt. Lett. 21, 1117 (1996). 